\title{
ON THE CHALLENGING VARIABILITY OF LS IV-14¹16: PULSATIONAL INSTABILITIES EXCITED BY THE $\epsilon$-MECHANISM
}

\author{
M. M. Miller Bertolami ${ }^{1,2}$, A. H. Córsico ${ }^{1,2}$, and L. G. Althaus ${ }^{1,2}$ \\ ${ }^{1}$ Facultad de Ciencias Astronómicas y Geofísicas, Universidad Nacional de La Plata, Paseo del Bosque s/n, 1900 La Plata, Argentina; \\ mmiller@fcaglp.unlp.edu.ar \\ 2 Instituto de Astrofísica La Plata (CCT-La Plata, UNLP-CONICET), Paseo del Bosque s/n, 1900 La Plata, Argentina \\ Received 2011 September 8; accepted 2011 September 22; published 2011 October 7
}

\begin{abstract}
We investigate the pulsation driving mechanism responsible for the long-period photometric variations observed in LS IV-14 116 , a subdwarf B star showing a He-enriched atmospheric composition. To this end, we perform detailed nonadiabatic pulsation computations over fully evolutionary post-He-core-flash stellar structure models, appropriate for hot subdwarf stars at evolutionary phases previous to the He-core burning stage. We found that the variability of LS IV- $14^{\circ} 116$ can be attributed to non-radial $g$-mode pulsations excited by the $\epsilon$-mechanism acting in the He-burning shells that appear before the star settles in the He-core burning stage. Even more interestingly, our results show that LS IV- $14^{\circ} 116$ could be the first known pulsating star in which the $\epsilon$-mechanism of mode excitation is operating. Last but not the least, we find that the period range of destabilized modes is sensitive to the exact location of the burning shell, something that might help in distinguishing between the different evolutionary scenarios proposed for the formation of this star.
\end{abstract}

Key words: stars: evolution - stars: individual (LS IV-14116) - stars: oscillations - subdwarfs

Online-only material: color figures

\section{INTRODUCTION}

About $5 \%$ of all hot subdwarf stars (sdB, sdO) show He-enriched surface abundances (He-sdB, He-sdO). While most normal H-rich subdwarfs are supposed to be low mass core He-burning stars with atmospheres dominated by $\mathrm{H}$ due to the action of gravitational settling, the evolutionary status of the He-rich subclass is less clear. He-rich subdwarfs have been suggested to be the result of either He white dwarf mergers (Saio \& Jeffery 2000) or late helium core flashes (Brown et al. 2001). Regardless the particular scenario proposed for their formation, it is accepted that some of these stars are still contracting toward the He-core burning phase (extreme horizontal branch, EHB), as otherwise gravitational settling of the remaining $\mathrm{H}$ would have turned the star into an H-rich sdB star.

Two families of pulsators have been discovered within the H-rich sdB stars: the rapid pulsators (sdBVr; Kilkenny et al. 2010) discovered by Kilkenny et al. (1997) and the slow pulsators (sdBVs; Kilkenny et al. 2010) discovered by Green et al. (2003). While sdBVr stars show short pulsation periods $(\sim 80-400 \mathrm{~s})$ ascribed to radial modes and non-radial $p$-modes, sdBVs pulsations (with periods $\sim 2500-7000 \mathrm{~s}$ ) are associated to non-radial long-period $g$-modes. Pulsations in both groups of variable stars have been explained by the action of the $\kappa$-mechanism due to the partial ionization of iron group elements in the outer layers, where these elements are enhanced by the action of radiative levitation (Charpinet et al. 1997; Fontaine et al. 2003). While many sdBV stars are known, only one He-sdB star, LS IV-14 116 , has been found to be a pulsator (Ahmad et al. 2004; Ahmad \& Jeffery 2005). In fact, LS IV- $14^{\circ} 116$ is a very intriguing object: while its atmospheric parameters $\left(T_{\text {eff }}\right.$ and $g$ ) place it well within the sdBVr instability region (Naslim et al. 2010; Green et al. 2011), it displays periods typical of the sdBVs family of pulsators (Ahmad \& Jeffery 2005; Green et al. 2011). From a spectroscopical perspective, LS IV- $14^{\circ} 116$ is also an intriguing object, showing a mild He-enrichment $\left(n_{\mathrm{He}}=0.16\right)$ and very high excesses of zirconium, yttrium, and strontium in its atmosphere (Naslim et al. 2011). In this sense, both the He-enrichment and the heavy metal excesses might be related to the effects of ongoing diffusion before reaching diffusive equilibrium, thus placing the star in the preEHB phase. Up to now, the driving mechanism behind the longperiod pulsations of LS IV- $14^{\circ} 116$, as well as the absence of short-period pulsations, remains a mystery and the star poses a challenge to the theory of stellar pulsations (Fontaine et al. 2008). In this connection, the recent confirmation of both the multiperiodic variability and the $T_{\text {eff }}-g$ values for LS IV- $14^{\circ} 116$ (Green et al. 2011) strongly increase the enigma.

While most pulsations exhibited by pulsating stars (included those of sdBVs and sdBVr stars) are self excited through the classical $\kappa$-mechanism, no star has been discovered so far to be excited by the $\epsilon$-mechanism. The only possible exception are some oscillations in $\delta$ Scuti stars (Lenain et al. 2006) and PG 1159 stars (Kawaler et al. 1986; Córsico et al. 2009), but neither of these have been confirmed. In the $\epsilon$-mechanism, the driving is due to the strong dependence of nuclear burning rates on temperature. In the layers where nuclear reactions take place, thermal energy is gained at compression by the enhancement of nuclear energy liberation, while the opposite happens during the expansion phase (Unno et al. 1989).

In the present Letter we show that long-period $g$-mode pulsations, like those observed in LS IV-14 ${ }^{\circ} 116$, can be understood as driven by the $\epsilon$-mechanism acting during the unstable shell burning events that take place before the star settles in the He-core burning phase. In addition, our scenario might explain the intriguing absence of short-period $p$-mode pulsations in LS IV- $14^{\circ} 116$. In the light of our results, LS IV- $14^{\circ} 116$ would be the first star in which pulsations driven by the $\epsilon$-mechanism have been detected, thus being the first proof that the $\epsilon$-mechanism can indeed drive pulsations in stars. 


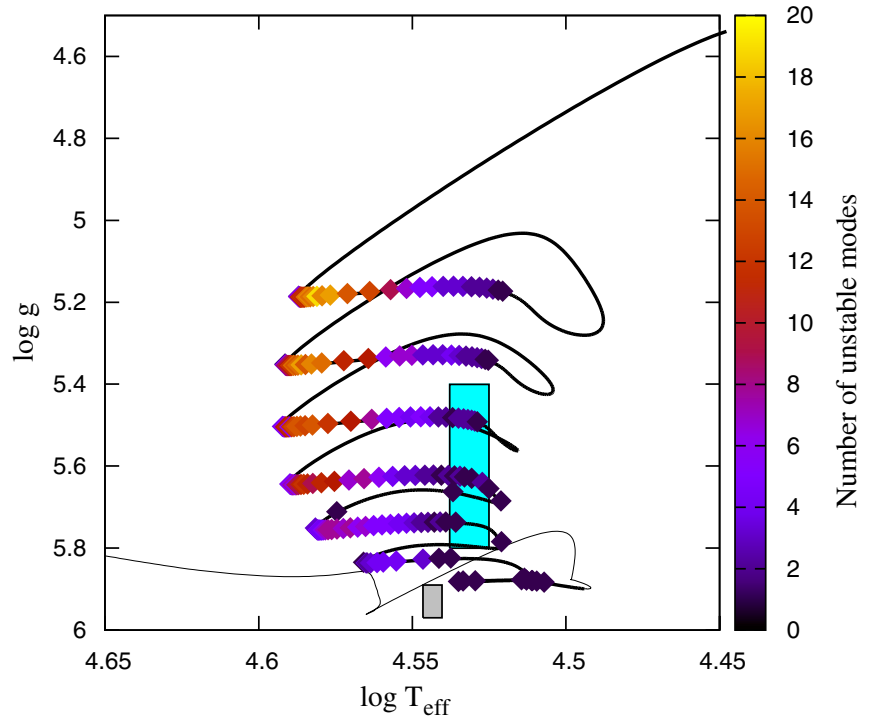

Figure 1. Evolution of our sequence before settling in the He-core burning phase. Thick line indicates pre-EHB evolution while thin line corresponds to the evolution after the EHB. Colored points indicate the location of models for which unstable modes have been found. These unstable modes are caused by the $\epsilon$-mechanism acting in the unstable He-burning subflashes that follow the primary He-core flash. Color coding indicates the number of unstable modes found in each model. Boxes indicate the location of LS IV-14 ${ }^{\circ} 116$ according to (Naslim et al. 2010; cyan) and (Green et al. 2011; gray).

(A color version of this figure is available in the online journal.)

\section{SCENARIO AND NUMERICAL DETAILS}

To explore the effects of the $\epsilon$-mechanism in a contracting pre He-core burning subdwarf star, we have computed the evolution of an initially $1.03 M_{\odot}, Z=0.02$ star model (see Miller Bertolami et al. 2008 for details about this sequence) that departs from the red giant branch just before the He-core flash, and experiences the flash as a shallow mixing hot flasher of $0.47378 M_{\odot}$ (mild He-enrichment, $Y=0.5978$; see Lanz et al. 2004 for a definition). Stellar structure and evolution equations have been solved with LPCODE stellar evolution code as in our previous works (Miller Bertolami et al. 2008 and references therein). Although our computations have been performed within the hotflasher scenario, the existence of He-shell subflashes in both the hot-flasher and merger scenarios makes our approach valid regardless of the actual origin of LS IV- $14^{\circ} 116$.

As seen in Figure 1, after the primary He-core flash (not shown in the figures) the star contracts toward the He-core burning phase through a series of He-shell subflashes (loops in Figure 1), evolving through the region in the $T_{\text {eff }}-g$ diagram occupied by LS IV- $14^{\circ} 116$. For this sequence we analyzed the pulsational stability properties of more than 1000 stellar structure models, one every five stellar evolution time steps, covering the whole evolution from the primary He-core flash to the final settling in the He-core burning phase. For each model we restricted ourselves to explore the $\ell=1 \mathrm{~g}$ modes in the period range from 500 to $6000 \mathrm{~s}$. The stability analysis of the stellar models was carried out by means of the linear, non-radial, nonadiabatic pulsation code described in Córsico et al. (2006) with the inclusion of the $\epsilon$-mechanism mode driving operating in He-burning layers as described in Córsico et al. (2009). Two standard simplifications have been introduced. We have adopted the "frozen-in convection" approximation in the stability analysis and assumed that $d S / d t=0$ in the non-perturbed background model adopted in the computa-
Table 1

Typical Timescales during the He-subflashes before the Begining of the EHB

\begin{tabular}{lccc}
\hline \hline No. of Subflash & $\begin{array}{c}\tau_{\text {duration }} \\
(\mathrm{yr})\end{array}$ & $\begin{array}{c}\text { Shortest } \tau_{\mathrm{e}} \\
(\mathrm{yr})\end{array}$ & $\begin{array}{c}\tau_{\text {glo }} \\
(\mathrm{s})\end{array}$ \\
\hline 1 & $\sim 2015$ & 110 & 31856 \\
2 & $\sim 4674$ & 250 & 33928 \\
3 & $\sim 7978$ & 610 & 46402 \\
4 & $\sim 11923$ & 1430 & 63176 \\
5 & $\sim 18286$ & 1970 & 85172 \\
6 & $\sim 23300$ & 2000 & 129910 \\
7 & $\ldots$ & $\ldots$ & 241674 \\
\hline
\end{tabular}

Notes. $\tau_{\text {duration }}$ indicates the length of the unstable stages during each $\mathrm{He}$ subflash, as shown in Figure 2. $\tau_{\mathrm{e}}$ is the $e$-folding time obtained from the nonadiabatic pulsation computations. $\tau_{\mathrm{glo}}$ is a global estimation of the convective turnover time (see the text).

tions. While the latter is not rigorously true in contracting stellar models, the timescale of the He-subflashes $(\sim 1000-10,000 \mathrm{yr})$ is nonetheless much longer than the oscillation periods (500-6000 s), and this approximation is correct. To assess the validity of "frozen-in convection" approximation, we computed the turnover timescale of convection at the peak of the helium flashes as

$$
\tau_{\mathrm{glo}}=\int_{R_{\text {base }}}^{R_{\mathrm{top}}} \frac{d r}{v_{\mathrm{MLT}}}
$$

where $R_{\text {base }}$ and $R_{\text {top }}$ are the radial coordinates of the boundaries of the convective zone and $v_{\text {MLT }}$ is the velocity of the convective elements within the mixing length theory of convection. As seen in the fourth column of Table 1, the convective turnover times are much longer than the unstable periods obtained in this work (see Figure 2). Thus, the assumption of "frozen-in convection" is largely justified.

\section{RESULTS}

In Figure 1 we can see how the star becomes recurrently a pulsator as it evolves toward the EHB. Our sequence of models is very close to the location of LS IV- $14^{\circ} 116$ in the $T_{\text {eff }}-g$ diagram. Note that no attempt to fit the exact location has been done, as we have just used a sequence from our previous work (Miller Bertolami et al. 2008). It would be easy to attain a better agreement by considering a sequence with a lower $\mathrm{He}$-abundance or earlier He-core flash (see Miller Bertolami et al. 2008). Figure 2 shows the evolution of the pulsation periods of our He-sdB sequence of models. In the upper panel we depict the situation found for the complete pre-EHB phase. Note that, each time a subflash develops, we obtain unstable $g$-modes, with the periods getting shorter as the star experiences subsequent, deeper, subflashes. The bottom panel illustrates the situation for the first and strongest flash of the series and shows the derived $e$-folding times for each mode. Notably, modes are continuously destabilized during the whole flash, with $e$-folding times $\left(\tau_{e}\right)$ of the excited modes getting shorter than the duration of the pulse $\left(\tau_{\text {duration }}\right)$, indicating that these modes should attain observable amplitudes (see also Table 1).

While the whole evolution from the primary He-flash to the EHB takes about $2 \mathrm{Myr}$, the unstable stages add up to $\sim 68,000 \mathrm{yr}$ (see Table 1). It is worth noting that this refers to the formally unstable stage, including some stages with somewhat long $e$-folding times, and that the actual unstable phases might be shorter. Then, within this picture, we would expect the variable 

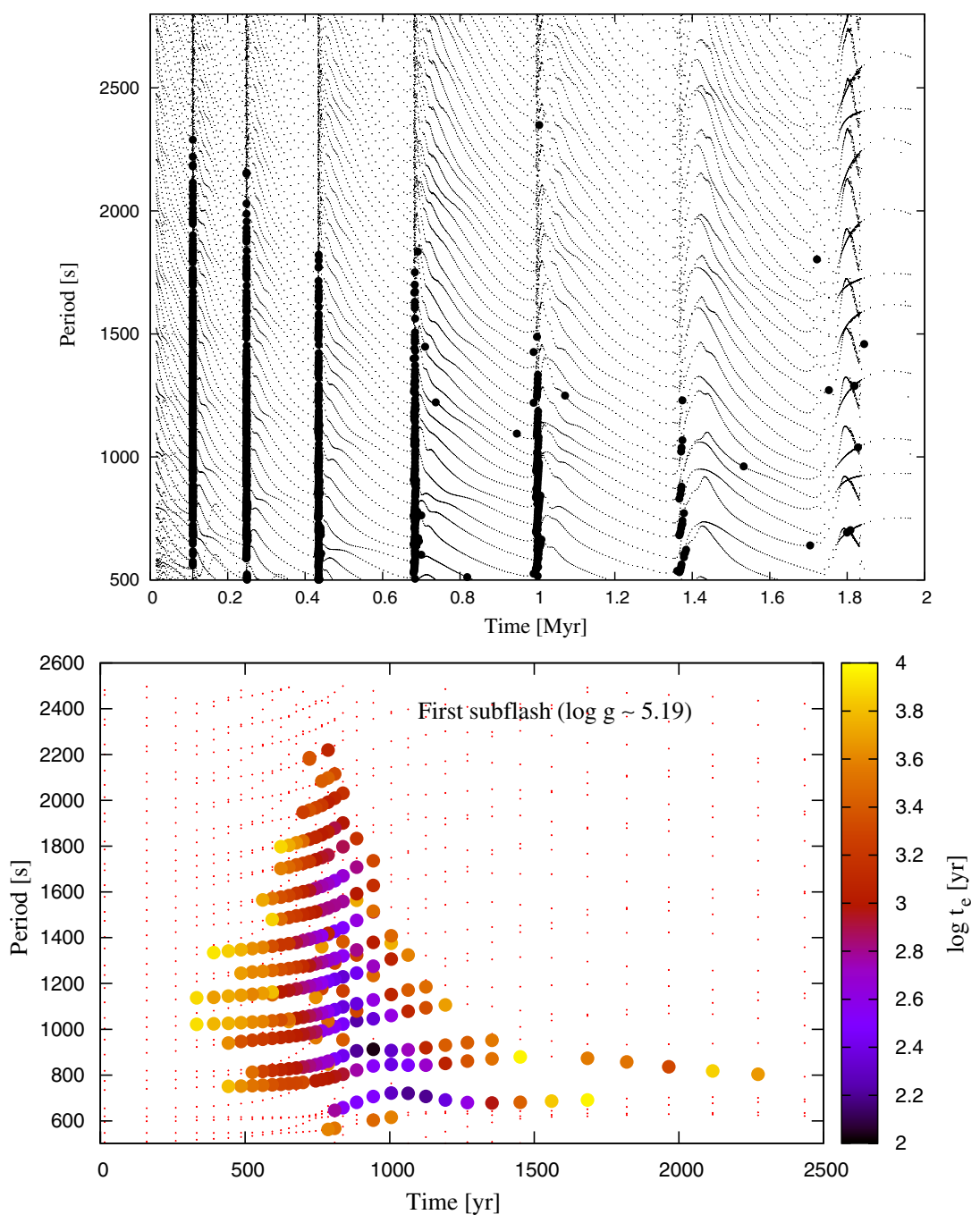

Figure 2. Upper panel: temporal evolution of the pulsation periods of our He-sdB models during the He-subflashes before the EHB. Thick points indicate those periods found to be formally unstable in our computations. Lower panel: the situation for the first and most intense subflash. Colored points indicate unstable modes with color coding indicating the $e$-folding time of each unstable mode.

(A color version of this figure is available in the online journal.)

to non variable ratio of $\mathrm{He}$-sdB to be lower than $1 / 30$. It is worth noting that this is consistent with the fact that only one He-sdB star, out of 23 studied objects, has been found to be a variable (Ahmad \& Jeffery 2005; Ahmad et al. 2004). As shown in Figure 2, most unstable modes exhibit periods in the range 600-2000 s close to the periods observed in LS IV-14 ${ }^{\circ} 116$ (1954-5084 s), and definitely much longer than the periods displayed by sdBVr stars in the same region of the $T_{\text {eff }}-g$ plane. In particular, it is worth noting that our models are able to reproduce the dominant periodicity observed in LS IV-14 ${ }^{\circ} 116$ $(P=1954 \mathrm{~s})$.

Our computations show that not all modes with periods within the unstable range are excited. In fact, only modes with significant amplitudes in the He-burning shell are destabilized. This can be seen in Figure 3 where the real part of the eigenfunction $\delta T / T$ and the differential work $(d W / d r)$ for two modes with consecutive radial orders $(k=26$; stable and $k=25$; unstable) are shown. This behavior, in which the $\epsilon$-mechanism acts as a narrowband filter exciting only modes with large amplitudes in the narrow He-burning shell region, has been reported in previous works (Kawaler et al. 1986; Córsico et al. 2009).
It has been mentioned (Green et al. 2011) that the effect of the enhancement of $\mathrm{He}$ in nonadiabatic computations in the context of the $\kappa$-mechanism in sdB stars might be related with the pulsations observed in LS IV- $14^{\circ} 116$. As a consequence, one might wonder as to what extent our results are related with the $\kappa$-mechanism. Also, one might wonder as to what extent the so called convective blocking mechanism (Pesnell 1987) at the He-shell flash convective zone might help in destabilizing the modes. In order to check our results, we have carried out explicit tests by switching off the derivatives of $\epsilon_{\mathrm{He}}$ in our nonadiabatic computations. As expected, when the $\epsilon$-mechanism is turned off, all unstable modes shown in Figure 2 become stable, indicating that the driving mechanism behind the unstable modes is indeed the $\epsilon$-mechanism alone.

As mentioned in previous paragraphs, our results suggest that the periods of unstable modes become shorter when the star experiences deeper subflashes, that is, when the burning shells move toward the core. One might then wonder where should the shell be located in order to excite modes with periods embracing the whole range of periods observed in LS IV-14 ${ }^{\circ} 116$. In this connection, we performed a numerical experiment by artificially shifting outward the location of the 


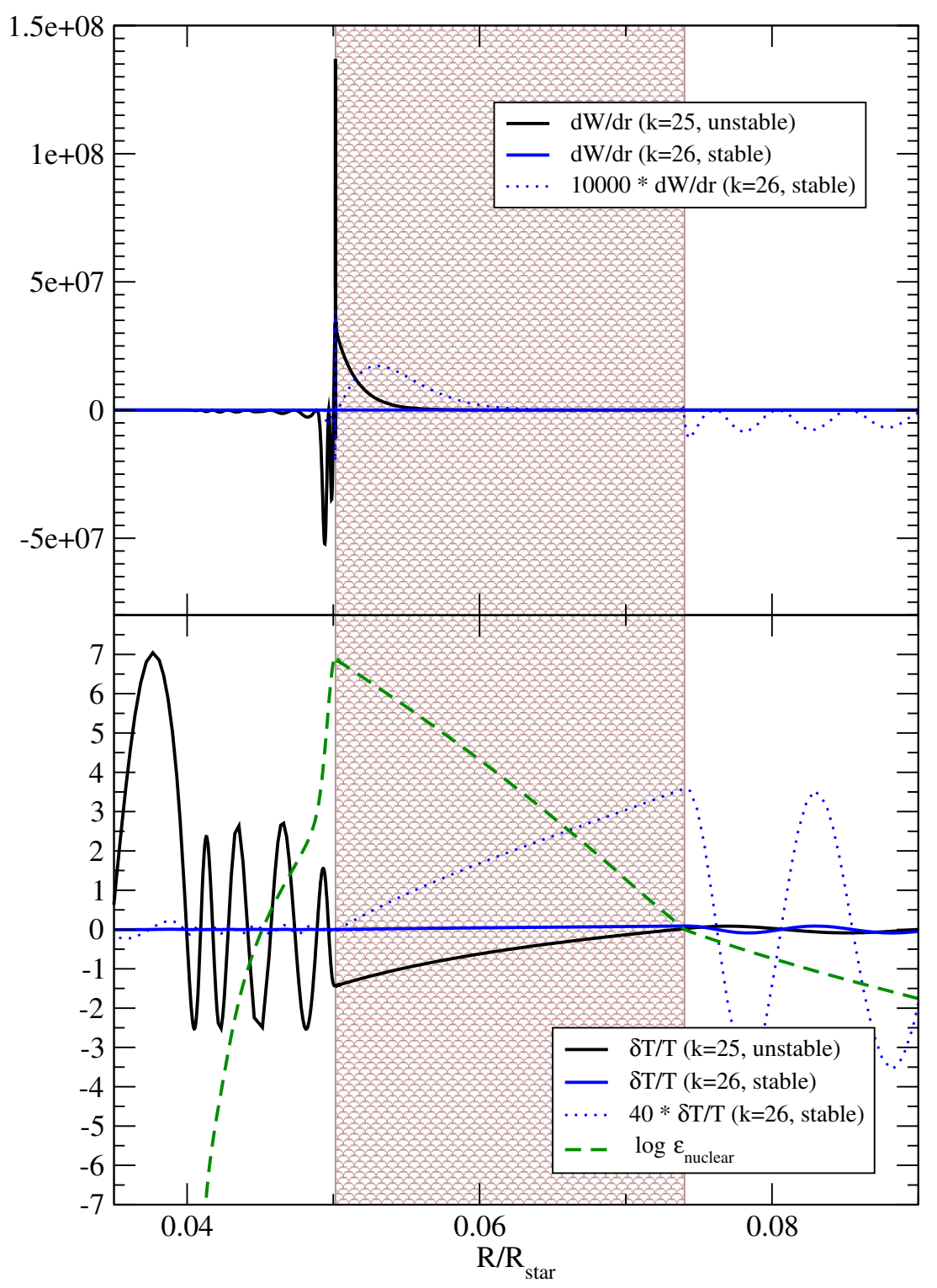

Figure 3. Lower panel: values of $\delta T / T$ for two consecutive modes during the first He-subflash, $k=25$ (unstable) and $k=26$ (stable). For the unstable mode, the large amplitude of $\delta T / T$ in the region of the He-burning shell can be easily appreciated. Upper panel: differential work $(d W / d r)$ for the modes $k=25$ and $k=26$. Note the strong driving contribution of the burning shell $(d W / d r>0)$ in the case of the unstable mode. In the interest of comparison we have arbitrarily magnified $\delta T / T$ and $d W / d r$ for the stable mode (dotted line). Brown curls indicate the location of the He-flash driven convective zone.

(A color version of this figure is available in the online journal.)

burning shell in our nonadiabatic computations. As shown in Figure 4, it would be enough to shift the location of the shell to $\log q=\sim-0.37\left(q:=1-m(r) / M_{\star}\right)$ in order to excite modes with periods covering the complete range of observed periods of LS IV-14 116 . Interestingly enough, this location is completely within the range of locations of the burning shells in the merger scenario (see Figures 2 and 3 of Saio \& Jeffery 2000). Whether this means that LS IV- $14^{\circ} 116$ is a post-merger object or that He-shell subflashes develop differently from that current stellar evolution models predict (as suggested by recent three-dimensional hydro-simulations of the event; Mocák et al. 2009) should be the object of further study.

\section{CONCLUSIONS}

We have shown that the $\epsilon$-mechanism is able to excite pulsations in the He-sdB star LS IV- $14^{\circ} 116$. Although our computations have been performed within the hot-flasher scenario, the existence of He-shell subflashes in both the hot-flasher and merger scenarios makes our conclusions valid regardless of the actual origin of LS IV- $14^{\circ} 116$.

Our results strongly suggest that long-period $g$-mode pulsations exhibited by LS IV-14 116 are driven by the $\epsilon$-mechanism. If so, it is the first confirmation that the $\epsilon$-mechanism can drive pulsations in stars. Interestingly, if LS IV- $14^{\circ} 116$ is a star in a fast evolutionary phase then iron and nickel might not have had enough time to accumulate in the driving region and drive $p$-mode pulsations, thus explaining also the intriguing absence of these modes in LS IV-14 116 light curve.

The fact that our model covers only partially the range of periods observed in LS IV-14 116 might be related to the possibility that the star is a post-merger object, in which the locations of the He-flash shells are different from those of hotflasher objects. In fact, the numerical experiment performed in 


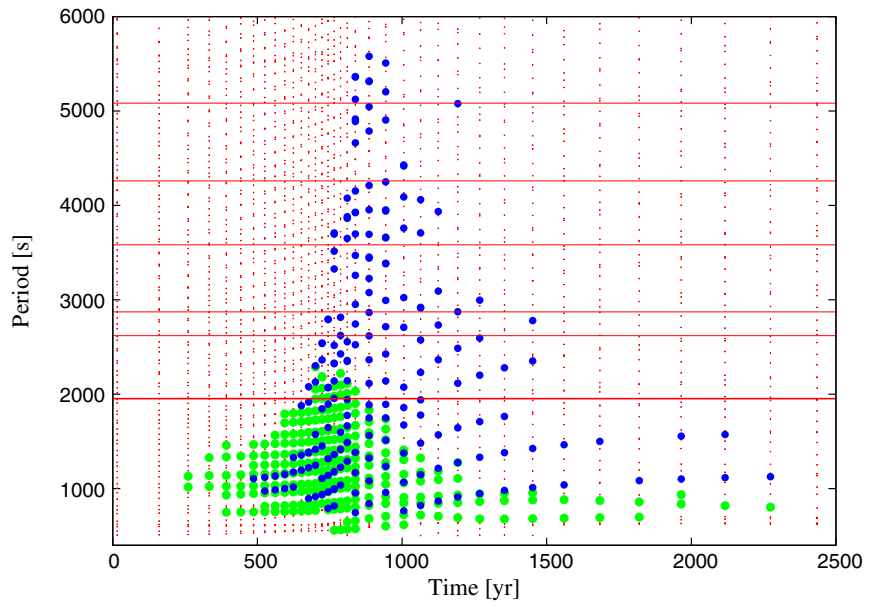

Figure 4. Effect of shifting the location of the burning shell on the periods of unstable modes in the first subflash. Blue points indicate modes destabilized when the burning shell is artificially shifted to $\log q \sim-0.37$. Green points show the unstable modes in our standard sequence (burning shell at $\log q \sim-0.21$; see Figure 3). Lines indicate the periods detected in LS IV $-14^{\circ} 116$, with their width being proportional to the amplitude of each mode (Green et al. 2011).

(A color version of this figure is available in the online journal.)

the previous section suggests that nonadiabatic computations of the $\epsilon$-mechanism on post-merger stellar models might show better agreement with the observed periods of LS IV-14 ${ }^{\circ} 116$. Computations of the $\epsilon$-mechanism in post-merger He-sdB stellar models would be very valuable.

In short, we have shown that the LS IV-14 ${ }^{\circ} 116$ atmospheric values $\left(T_{\text {eff }}, g\right.$, and $\left.n_{\mathrm{He}}\right)$, the existence of long-period $g$-mode pulsations, and possibly the absence of the classic $\kappa$-mechanism $p$-mode pulsations can be simultaneously explained if LS IV- $14^{\circ} 116$ is a star undergoing a He-shell flash on its way toward the EHB.

We thank the referee (Dr. Wojciech Dziembowski) for his valuable time and encouraging report. M3B also thanks both the Varsavsky Foundation and the organizers of the Fifth Meeting on Hot Subdwarf Stars \& Related Objects for the financial assistance that allowed him to attend the meeting, where the central idea of this article was conceived. This work has been supported by grants PIP 112-200801-00904 and PICT-2010-0861 from CONICET and ANCyT, respectively. This research has made use of NASA's Astrophysics Data System.

\section{REFERENCES}

Ahmad, A., \& Jeffery, C. S. 2005, A\&A, 437, L51

Ahmad, A., Jeffery, C. S., Solheim, J.-E., \& Østensen, R. 2004, Ap\&SS, 291 435

Brown, T. M., Sweigart, A. V., Lanz, T., Landsman, W. B., \& Hubeny, I. 2001, ApJ, 562, 368

Charpinet, S., Fontaine, G., Brassard, P., et al. 1997, ApJ, 483, L123

Córsico, A. H., Althaus, L. G., \& Miller Bertolami, M. M. 2006, A\&A, 458, 259

Córsico, A. H., Althaus, L. G., Miller Bertolami, M. M., González Pérez, J. M. \& Kepler, S. O. 2009, ApJ, 701, 1008

Fontaine, G., Brassard, P., Charpinet, S., et al. 2003, ApJ, 597, 518

Fontaine, G., Brassard, P., Charpinet, S., et al. 2008, in ASP Conf. Ser. 392, Hot Subdwarf Stars and Related Objects, ed. U. Heber, C. S. Jeffery, \& R. Napiwotzki (San Francisco, CA: ASP), 231

Green, E. M., Fontaine, G., Reed, M. D., et al. 2003, ApJ, 583, L31

Green, E. M., Guvenen, B., O’Malley, C. J., et al. 2011, ApJ, 734, 59

Kawaler, S. D., Winget, D. E., Hansen, C. J., \& Iben, I., Jr. 1986, ApJ, 306, L41

Kilkenny, D., Fontaine, G., Green, E. M., \& Schuh, S. 2010, Inf. Bull. Var. Stars, 5927, 1

Kilkenny, D., Koen, C., O’Donoghue, D., \& Stobie, R. S. 1997, MNRAS, 285, 640

Lanz, T., Brown, T. M., Sweigart, A. V., Hubeny, I., \& Landsman, W. B. 2004, ApJ, 602, 342

Lenain, G., Scuflaire, R., Dupret, M.-A., \& Noels, A. 2006, Commun. Asteroseismol., 147, 93

Miller Bertolami, M. M., Althaus, L. G., Unglaub, K., \& Weiss, A. 2008, A\&A, 491, 253

Mocák, M., Müller, E., Weiss, A., \& Kifonidis, K. 2009, A\&A, 501, 659

Naslim, N., Jeffery, C. S., Ahmad, A., Behara, N. T., \& Şahìn, T. 2010, MNRAS, 409,582

Naslim, N., Jeffery, C. S., Behara, N. T., \& Hibbert, A. 2011, MNRAS, 412, 363

Pesnell, W. D. 1987, ApJ, 314, 598

Saio, H., \& Jeffery, C. S. 2000, MNRAS, 313, 671

Unno, W., Osaki, Y., Ando, H., Saio, H., \& Shibahashi, H. 1989, Nonradial Oscillations of Stars (2nd ed.; Tokyo: Univ. Tokyo Press) 